\title{
Авангард мирового аналитического оборудования
}

\author{
Е.Н. Обухова, К.х.н., \\ ФГБУ ГНЦ ИнстИтут ИммунологиИ ФМБА России \\ enobukhva@yandex.ru
}

УдК: 543.07

\begin{abstract}
Ежегодно в мире проходит огромное количество выставок и конференций, некоторые из них представляют особый интерес для специалистов в области аналитической химии, поскольку определяют направления развития отрасли. Два таких мероприятия прошли весной-летом 2019 года в США: Питтсбургская конференция по аналитической химии и прикладной спектроскопии и ежегодная Конференция Американского массспектрометрического общества. Тысячи ученых посетили конференции и следили за их программами в интернет-трансляциях, а сопутствующие выставки собрали ведущих производителей аналитического оборудования и материалов.
\end{abstract}

\section{THE PITTCON TODAY EXCELLENCE AWARDS: ИННОВАЦИИ, МЕНЯЮЩИЕ МИР}

Юбилейная 70-я Питтсбургская конференция по аналитической химии и прикладной спектроскопии (Pittcon, Pittsburg Conference on Analytical Chemistry and Applied Spectroscopy) состоялась 17-21 марта 2019 года в Филадельфии. Конференция считается наиболее значимым и посещаемым ежегодным событием в области аналитической химии и спектроскопии в мире и сопровождается масштабной выставкой лабораторных материалов, оборудования, программного обеспечения и систем контроля.

Pittcon начинался в 1950 году как небольшая техническая конференция. За последующие 18 лет его популярность так выросла, что отели Питтсбурга перестали вмещать всех желающих. Тогда конференцию стали проводить в других, более крупных городах, а название осталось прежним. В 2019 году Pittcon посетили 12500 человек из 94 стран, 713 компаний представили свою продукцию на 1234 стендах. В программу конференции вошли более 2000 презентаций, многочисленные семинары, 75 обучающих курсов.

Ключевое событие конференции - вручение премии The Pittcon Today Excellence Awards. Ежегодно сотни компаний представляют на Pittcon новинки аналитического приборостроения и Life Science. Отбирает финалистов независимое жюри, состоящее из специалистов в области образования, промышленности и торговли. Оцениваются оригинальность идей, исполнение, результаты и прогнозируемое влияние на отрасль. Призы присуждают в трех категориях, в зависимости от объемов продаж, заявленных компаниями: до 10 млн, от 10 до 100 млн, свыше 100 млн долл.

\section{Категория "До 10 млн долл."}

Второй год подряд золото в этой номинации получает компания Gate Scientific за магнитные мешалки со встроенными датчиками рН и температуры. Штаб-квартира и производство Gate Scientific располагаются в Силиконовой долине, основное направление деятельности - разработка и выпуск новых продуктов для лабораторной диагностики и Life Science. В прошлом году компания представила магнитные мешалки smartSENSE для контроля температуры жидкости, а в этом - модель усовершенствовали: в стержни мешалок добавили рН-чувствительный элемент и электрод сравнения.

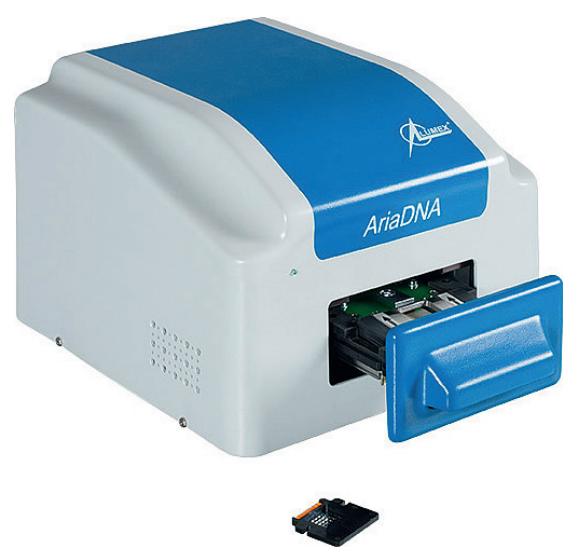

Puc. 1.

Микрочиповый ПЦР-анализатор AriaDNA, Lumex 


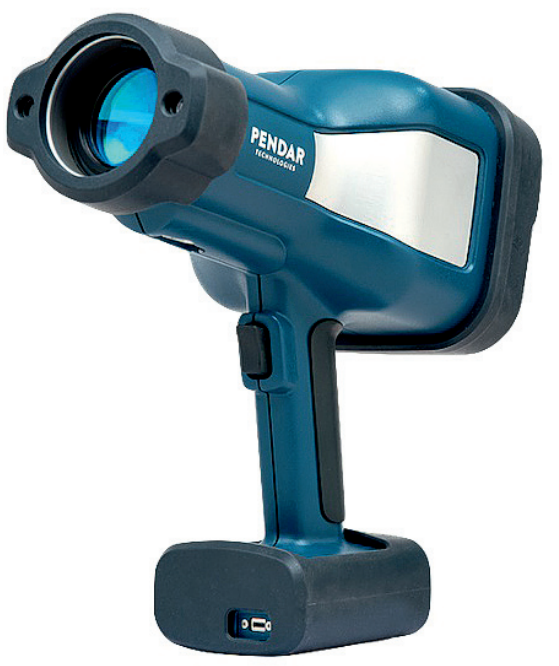

Puc. 2.

Портативный рамановский анализатор Pendar X10, Pendar Technologies

SmartSENSE оснащены сенсорами на основе идентификации радиочастот (radio-frequency identification) и непрерывно передают информацию в беспроводную лабораторную станцию, которая в режиме реального времени регулирует нагрев для поддержания заданной температуры, скорость перемешивания и сохраняет значения рН. Управлять станцией можно удаленно с компьютера или смартфона. С помощью SmartSENSE можно готовить буферы для хроматографии, титровать, измерять скорость реакции, наблюдать за изменением состава и протеканием биопроцессов в жидкой среде, в том числе в герметичных емкостях. Компания работает над расширением ассортимента беспроводных датчиков и разрабатывает систему беспроводной зарядки мешалок с использованием магнитного поля. В будущем SmartSENSE может стать альтернативой лабораторным химическим реакторам.

Серебро выиграл высокоскоростной ПцР-анализатор на основе микрочипов AriaDNA компании Lumex Instruments (рис. 1). С помощью ПЦР-анализаторов нарабатывают фрагменты последовательностей нуклеиновых кислот из биологических проб с последующим детектированием. Для этого пробу помещают в смесь реагентов (ПЦР-смесь) и повторяют циклы нагрев-охлаждение, чтобы регулировать работу ферментов, которые копируют и синтезируют нужные участки ДНК или РНК. В ПЦР-анализаторе AriaDNA пробы анализируются на микрочипах на 20 или 40 лунок объемом 1,2 мкл. Благодаря малому объему лунки быстро нагреваются и охлаждаются (10-12 $\left.{ }^{\circ} \mathrm{C} / \mathrm{c}\right)$, и за 20 мин прибор совершает 45 ПЦР-циклов, детектируя до 1-5 копий ДНК/ РНК в пробе. В лунки микрочипов нанесены готовые лиофилизированные ПЦР-смеси, что исключает необходимость приготовления растворов. По сравнению с аналогичными приборами AriaDNA работает в три раза быстрее, что существенно снижает стоимость анализа. К прибору предлагаются готовые диагностические наборы для определения вирусных и бактериальных инфекций, контроля происхождения продуктов питания и биологической безопасности. Компактный,

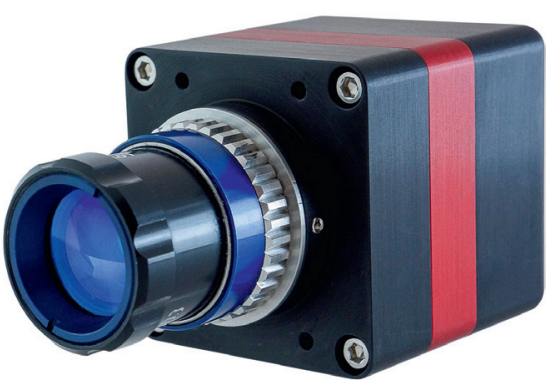

Puc. 3. Камера OWL 1280, Raptor Photonics

чувствительный, экспрессный ПЦР-анализатор AriaDNA будет востребован в медицинских, ветеринарных, сельскохозяйственных, таможенных лабораториях.

Бронзовую награду получил портативный рамановский анализатор Pendar X10 (рис. 2). Это первый прибор, который компания Pendar Technologies представляет на рынке, и международное признание свидетельствует о высокой квалификации инженеров и качестве продукта. Компания использует технологию, разработанную и лицензированную в Университете Гарварда. Основные преимущества Pendar X10 по сравнению с аналогичными приборами включают: увеличенное расстояние до объекта исследования (до 1 м), минимальный риск возгорания светочувствительных веществ, высокую скорость анализа (темные и флуоресцентные материалы анализируются в течение 30 с, белые порошки - 10 с), безопасность для оператора (не требуется защита для глаз).

\section{Категория}

\section{"от 10 до 100 млн долл."}

Золотую награду получила камера видимого и коротковолнового инфракрасного диапазона OWL 1280 компании Raptor Photonics (рис. 3). Камеры, фиксирующие коротковолновое инфракрасное излучение, используются при слабом освещении и в экстремальных условиях и, как правило, снимают в диапазоне 0,9-1,7 мкм. Камера OWL 1280 с сенсорами на основе арсенида индия-галлия снимает в области 0,4-1,7 мкм. Шаг пикселя OWL 1280 составляет $10 \times 10$ мкм, камера имеет низкий шум считывания (менее 40 электронов) и сверхвысокий динамический диапазон - все это гарантирует высокое разрешение и чувствительность съемки. Камера OWL 1280 компактная, прочная, работает без охлаждения и характеризуется низким энергопотреблением (<3 Вт).

Серебро жюри присудило компании Tosoh Bioscience за аффинную хроматографическую колонку TSKgel FcR-IIIANPR. Полвека Tosoh Bioscience работает в области высокоэффективной жидкостной и эксклюзионной хроматографии синтетических и биополимеров. 30 лет назад была создана серия сорбентов NRP (Non-Porous Resins) для хроматографических колонок. Частицы сорбента состоят из метакрилатного пористого ядра, на которое нанесен слой непористого адсорбента. На основе сорбента NRP разработана 

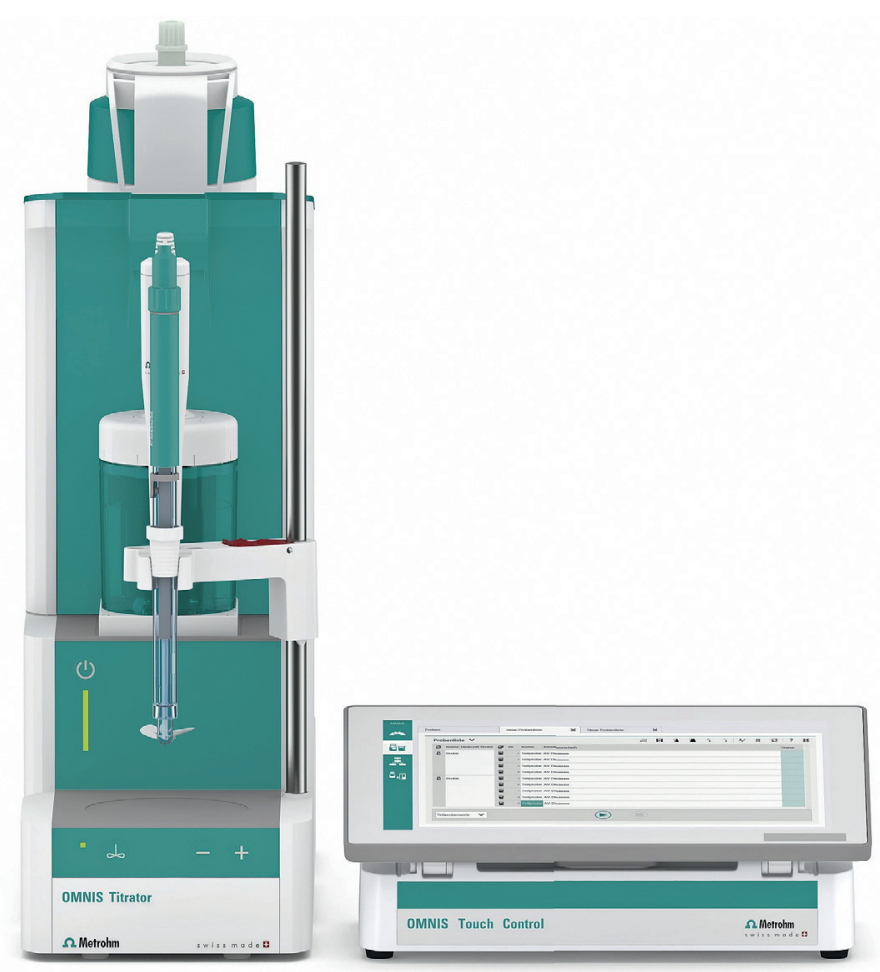

Puc. 4. Автоматический mumpamop OMNIS, Metrohm

аффинная колонка для оценки биологической активности моноклональных антител.

Использование моноклональных антител в терапии рака - актуальное направление современной медицины и биотехнологии. Основную роль в процессе антителозависимой цитотоксичности играет рецептор $\mathrm{F}_{\mathrm{c}}$ gamma IIIa на поверхности антител. Рецептор представляет собой гликозилированный белок, структура и количество его гликанов влияют на свойства антител, в том числе на противораковую активность. Колонка TSKgel FcR-IIIA-NPR заполнена NRP-сорбентом, на поверхности которого иммобилизован лиганд рецептора $F_{c}$ gamma IIIa. В первую очередь с колонки элюируются гликоформы, которые слабо связываются с сорбентом и, следовательно, имеют низкую антитело-зависимую цитотоксичность. Картина хроматографического разделения помогает соотнести особенности гликозилирования антител с противораковой активностью. На колонку можно наносить как очищенные образцы, так и супернатанты культуральных жидкостей, а время анализа смеси антител составляет 30 мин. Подобный экспрессный метод востребован на разных этапах биотехнологических производств: скрининг клеточных линий, сравнение биоаналогов и оригинальных препаратов, разработка и оптимизация производства, мониторинг биоинженерных исследований, контроль качества партий антител.

Бронзовую награду получила компания Metrohm за OMNIS - автоматический титратор по методу Карла Фишера (рис. 4) для определения воды в пробах. OMNIS имеет модульный дизайн и работает в четыре раза быстрее предыдущих моделей. Потребитель может добавить дополнительные интерфейсы или дозирующие модули и превратить титратор в автоматическую платформу для высокопроизводительного анализа. В максимальной комплектации OMNIS анализирует до четырех проб одновременно, и рассчитан на загрузку 175 образцов. Metrohm предлагает готовые протоколы и реагенты для анализа. Контейнеры для реагентов маркируются бар-кодами, которые содержат информацию о растворе и дате изготовления и автоматически считываются и регистрируются в программном обеспечении титратора и головном компьютере.

\section{Категория "Свыше 100 млн долл."}

SHIMADZU Scientific Instruments получил золотую награду за хроматограф Nexera UC для препаративной сверхкритической флюидной хроматографии (СФХ) (рис. 5). Прибор предназначен для препаративных разделений лабораторного масштаба в фармацевтических компаниях. Метод препаративной СФХ эффективен для разделения веществ массой до 10 кДа, в том числе хиральных изомеров. Однако выделить и собрать разделенные соединения было затруднительно: газ-носитель (углекислый газ) на выходе из колонки расширялся и уносил компоненты пробы в лабораторное

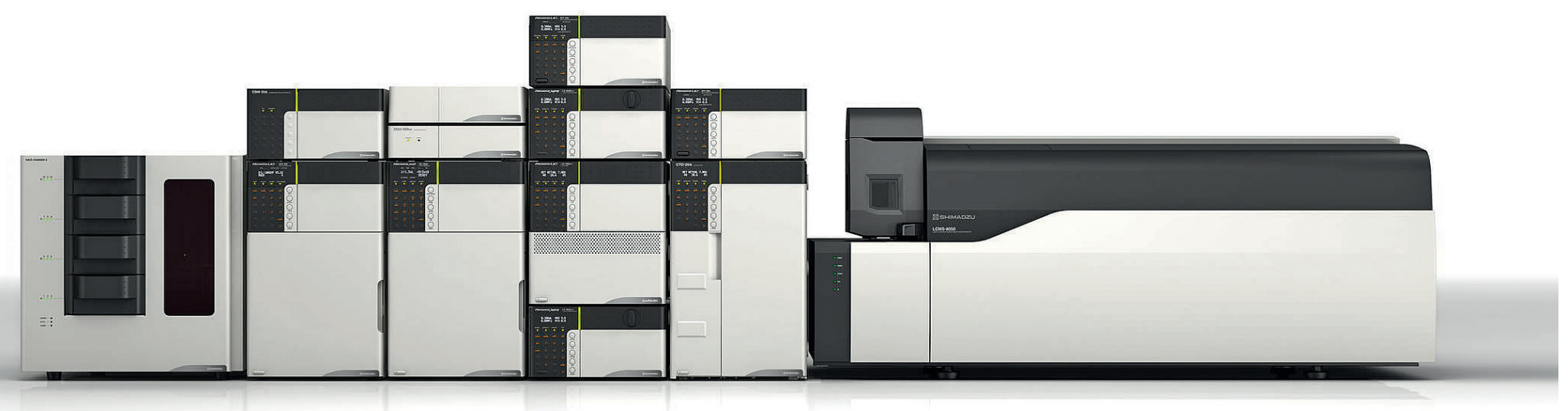

Puc. 5. Nexera UC, SHIMADZU Scientific Instruments 
пространство, что не только снижало выход продукта, но и было опасно для здоровья. Специалисты компании разработали газожидкостный сепаратор для сбора образцов, который решил эту проблему.

Компания Phenomenex, производитель комплектующих для газовой и жидкостной хроматографии, выиграл серебряную награду за колонку для газовой хроматографии Zebron ZB-624PLUS. PLUS в названии колонки обозначает новую, более инертную, неподвижную фазу с диапазоном рабочих температур до 300-320 ․ Колонка

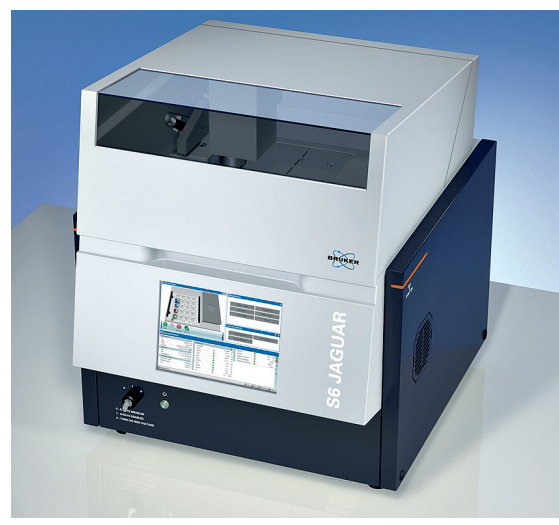

Puc. 6. SG JAGUAR, Bruker Inc

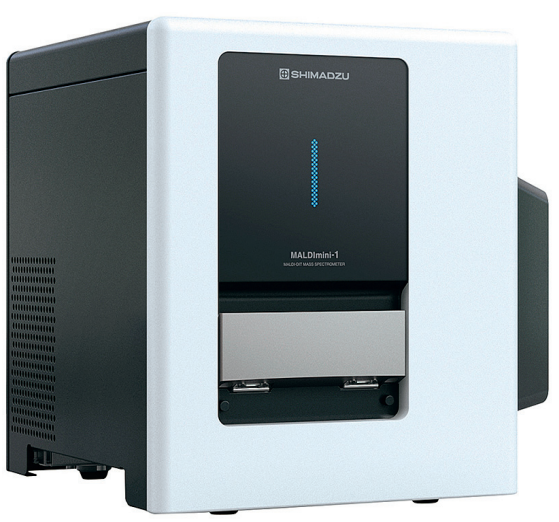

Puc. 7. MALDImini-7, SHIMADZU имеет низкую фоновую течь, что увеличивает чувствительность масс-спектрометрического детектирования. Умеренно полярная фаза подходит для анализа полярных и неполярных аналитов, особенно в растворителях с высокой температурой кипения.

Бронзу в этом году получил Bruker за настольный волнодисперсионный рентгенофлуоресцентный спектрометр S6 JAGUAR (рис. 6). Производительность прибора увеличена в два раза по сравнению с предыдущими моделями: мощности (400 Вт, 50 кВ) хватает для анализа тяжелых и легких элементов, четыре кристалла-анализатора охватывают широкий круг определяемых элементов, а рентгеновская оптика оптимизирована для снижения фонового сигнала.

The Pittcon Today Excellence Awards - это не только престижная награда, но и мировое признание представленных инноваций.

Следующая конференция Pittcon пройдет в Чикаго (США) с 1 по 5 марта 2020 года.

\section{7-Я КОНФЕРЕНЦИЯ АМЕРИКАНСКОГО МАСС-СПЕКТРОМЕТРИЧЕСКОГО ОБЩЕСТВА: БУДУЩЕЕ МАСС-СПЕКТРОМЕТРИИ СЕГОДНЯ}

67-я ежегодная конференция американского массспектрометрического общества прошла 2-6 июня 2019 года в Атланте. Крупнейшее событие в области масс-спектрометрии собрало посетителей со всего мира, а лидеры рынка массспектрометрического оборудования традиционно анонсировали на конференции новые приборы.

Американское масс-спектрометрическое общество (АMCO) - некоммерческая организация, образованная в 1969 году с целью распространения знаний о массспектрометрии и родственных областях знаний. Членами АМСО состоят более 8500 ученых из академических, промышленных и государственных лабораторий. Ежегодная конференция АМСО является знаковым событием в области масс-спектрометрии. Список тем конференции обширен: аналитическая и клиническая химия, биоинформатика,

обработка данных, метаболомика, протеомика, липидомика, анализ нуклеиновых кислот, фармакокинетика, анализ продуктов питания, нефтехимия, анализ объектов окружающей среды - все области науки, медицины, производства, где используют масс-спектрометрический анализ. В этом году в Конференции участвовали 6675 ученых из 54 стран, в научную программу вошли 3133 устных сообщений, лекций и стендовых докладов, 18 семинаров и четыре обучающие лекции. Производители приборов и представители промышленных, медицинских и научно-производственных компаний ждали посетителей на 180 стендах.

\section{MALDImini-1 - компактный масс-спектрометр} с цифровой ионной ловушкой

SHIMADZU объявила о выходе на рынок MALDImini-1, первого компактного масс-спектрометра с цифровой ионной ловушкой и источником матрично-вспомогательной лазерной десорбции и ионизации (MALDI) (рис. 7). В основе уникальной технологии - замена синусоидальных радиочастотных волн на прямоугольные. Настройка частоты волн в цифровой ионной ловушке не требует высокого напряжения, поэтому прибор работает от обычного источника переменного тока. При весе 25 кг MALDImini-1 занимает место размером с лист бумаги формата АЗ. Лазерно-оптическая система, пробоотборник и вакуумная вытяжка оптимизированы для максимальной компактности. Цифровая ионная ловушка захватывает ионы с массой до 70000 Да и функционирует в режиме тандемной масс-спектрометрии (МС / MC и МС ${ }^{3}$ фрагментация), что в сочетании с источником ионизации MALDI позволяет анализировать широкий круг биомолекул: от небольших пептидов и гликанов до высокомолекулярных соединений.

\section{SpatialOMx -}

два источника ионизации в одном

Компания Bruker представила на конференции массспектрометр SpatialOMx с двумя встроенными источниками ионизации: электрораспыления (ESI) и MALDI (рис. 8). Прибор сконструирован на основе последней модели 


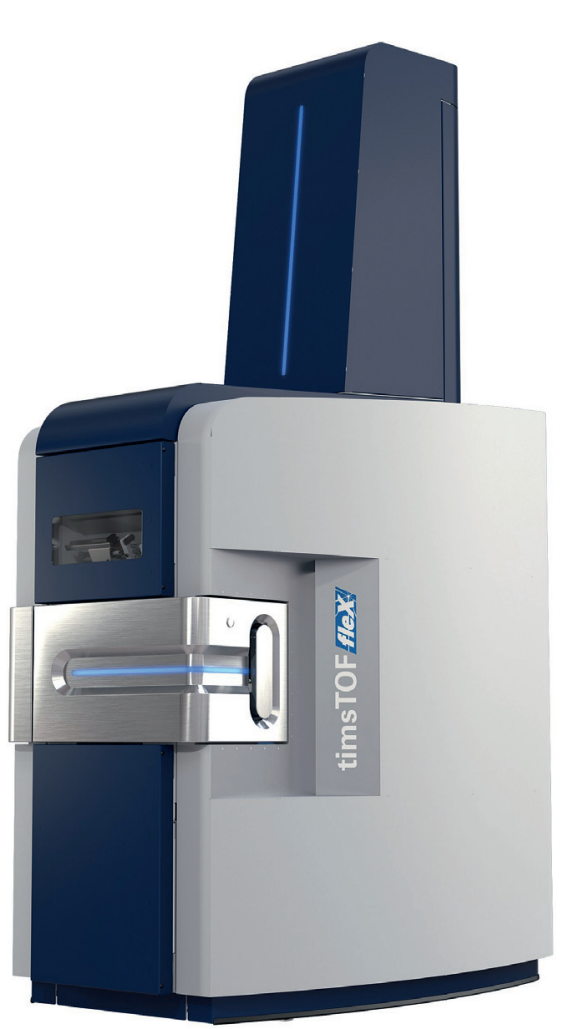

Puc. 8. SpatialOMx, Bruker

времяпролетного масс-спектрометра timsTOF fleXTM с разделением по ионной мобильности.

Распространенная проблема протеомики состоит в том, что при пробоподготовке смешиваются клетки из разных клеточных популяций, результаты анализа усредняются и теряется информация о биохимических и патологических процессах. Благодаря совместному использованию ESI и MALDI можно изучать препараты живых тканей и смесей клеток и получать многомерную картину распределения интересующих соединений. На первом этапе SpatialOMx анализирует пробу с MALDI-ионизацией и показывает пространственное распределение клеточных популяций. На втором этапе с использованием ESI сравнивают качественный и количественный состав интересующих соединений (пептидов, гликанов, липидов, белков, ксенобиотиков) в заданных типах клеток. Программа переключает источники в соответствии с условиями пользователя, автоматически аннотирует детектированные вещества и выделяет релевантную информацию. SpatialOMх будет востребован в исследовании раковых заболеваний, когда невозможно детектировать малые концентрации биомаркеров традиционными методами.

\section{Echo MS: одна капля - один анализ}

На стенде компании SCIEX посетители конференции наблюдали будущее высокопроизводительного массспектрометрического анализа - Acoustic Ejection Mass Spectrometry (AEMS, масс-спектрометрия звукового выталкивания). AEMS представляет собой систему прямого ввода в масс-спектрометр на основе двух разработок. Компания Labcyte создала технологию звукового выброса капли для точного дозирования нанолитров жидкости без использования пипетки. Специалисты SCIEX разработали открытый порт для отбора проб, чтобы напрямую переносить капли в масс-спектрометр. Интерфейс прибора состоит из двух коаксиальных трубок: растворитель течет в полости между трубками, достигает конца и всасывается потоком газараспылителя во внутреннюю трубку, захватывая каплю образца. Капля переносится в источник ионизации ESI и анализируется в масс-спектрометре Sciex 5500. Система непрерывно самоочищается и работает без жидкостного хроматографа и хроматографической колонки.

Ключевой параметр в AEMS - скорость потока растворителя - устанавливается экспериментально и гарантирует воспроизводимость дозирования и узкие, симметричные масс-спектрометрические пики. В ходе тестирования система отбирала пробы объемом 2,5 нл со скоростью до трех капель в секунду, сохраняя высокую точность и воспроизводимость количественного анализа тестовых веществ.

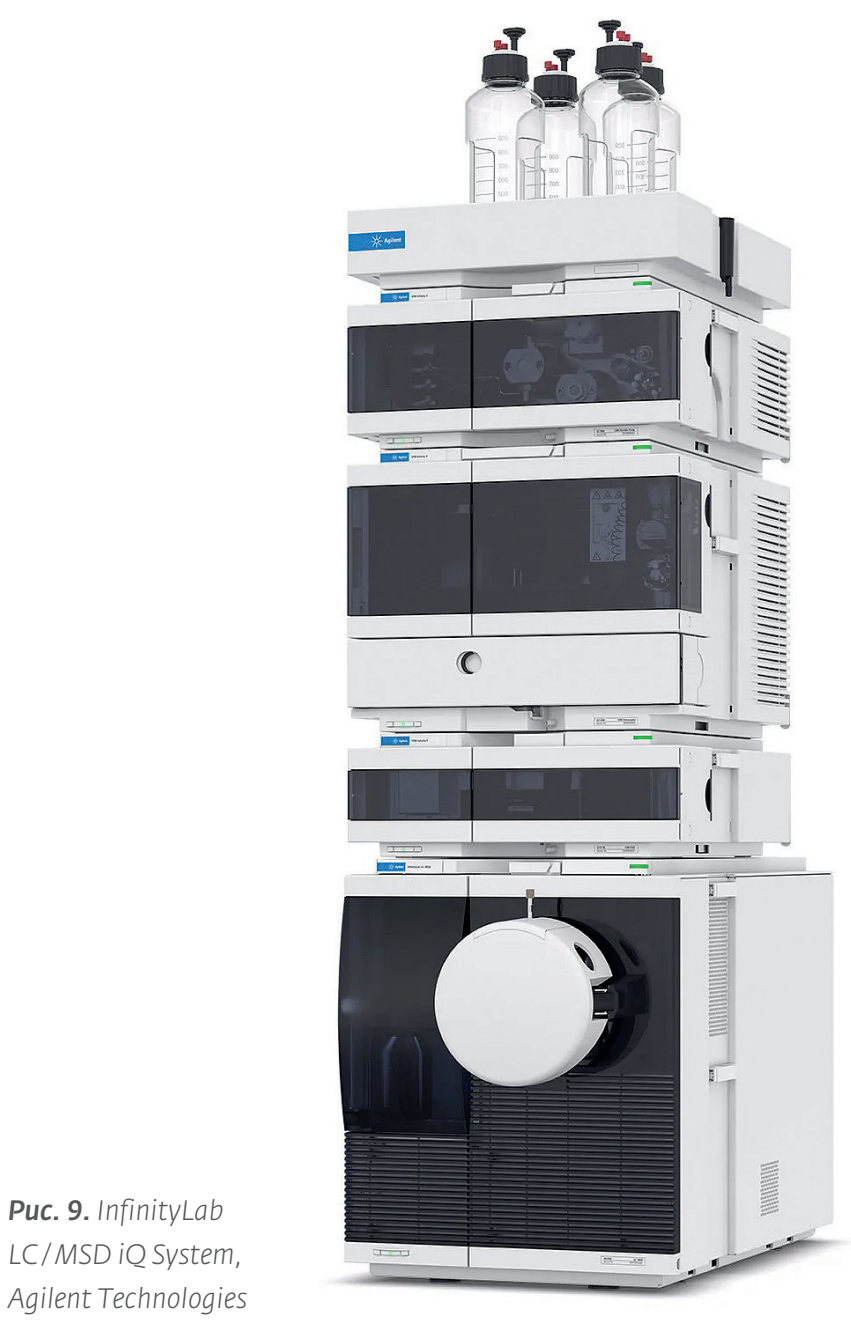




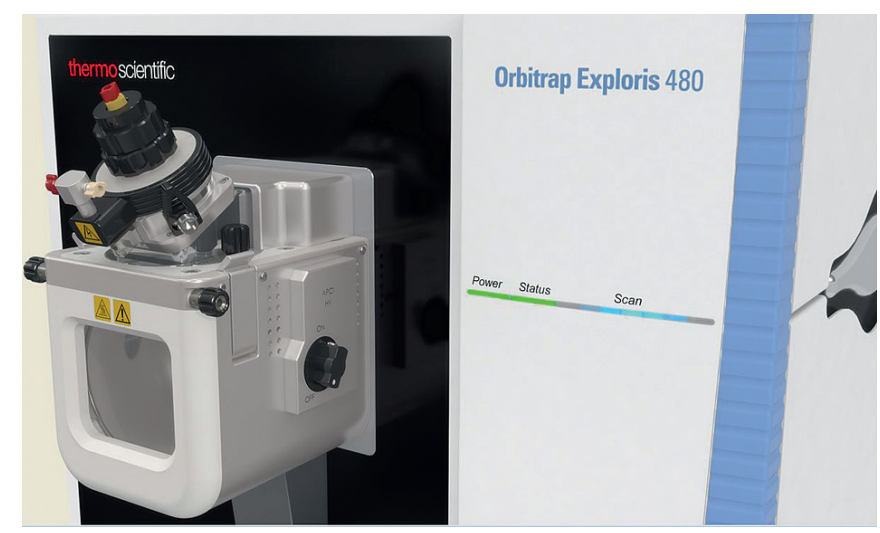

Puc. 10. Orbitrap Exploris 480 MS, Thermo Fisher Scientific

На стенде SCIEX посетители могли увидеть прототип масс-спектрометра Echo MS на основе технологии AEMS. Прибор предназначен для скрининга проб в фармацевтическом и биотехнологическом производствах и по оценкам инженеров SCIEX способен сократить время анализа со 100 дней до нескольких суток.

Проще, быстрее, надежнее

Компании Agilent и Thermo Fisher Scientific представили свои новинки - надежные и производительные хроматомасс-спектрометры.

InfinityLab LC/MSD iQ System пополнил линейку жидкостных хроматографов - масс-спектрометров Agilent Technologies (рис. 9). Прибор оборудован системой проверки работоспособности (health-check system), которая контролирует текущее состояние, дает обратную связь при обслуживании и поддерживает бесперебойную работу. Встроенные сенсоры собирают и отображают информацию о статусе, готовности и конфигурации. Для одновременной проверки пригодности хроматографа и масс-спектрометра подобрана специальная тестовая смесь. Модульная конструкция экономит лабораторное пространство и облегчает замену растворителей и деталей. Функция обратной связи при техническом осмотре помогает планировать замену расходных материалов в соответствии с графиком лаборатории, что повышает производительность. Программное обеспечение Agilent OpenLab CDS собирает данные и создает отчеты в автоматическом режиме, экономя время пользователя. Масс-спектрометр InfinityLab Flex Bench MS оборудован системами снижения шума и управления отходами.

Новинка компании Thermo Fisher Scientific - компактный масс-спектрометр высокого разрешения Orbitrap Exploris 480 MS (рис. 10). Инструмент предназначен для трансляционных исследований и высокопроизводительного количественного анализа белков и малых молекул в биофармацевтических и протеомных лабораториях. Для этих целей в приборе улучшены показатели количественного анализа без меток и расширены возможности
PUc. 11. SELECT

SERIES CyClic IMS, Waters

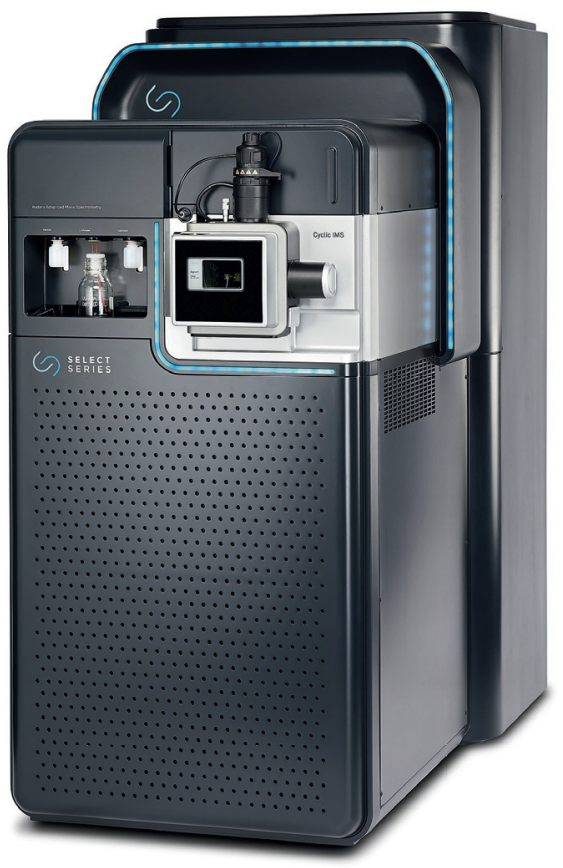

мультиплексного определения белков в сложных биологических матрицах. Orbitrap Exploris 480 MS имеет меньшие размеры и проще в обслуживании, чем предыдущие модели.

\section{SELECT SERIES Cyclic IMS:}

ионы бегают по кругу

Компания Waters представила на конференции новый масс-спектрометр с разделением по ионной подвижности SELECT SERIES Cyclic IMS (рис. 11). Традиционно регион разделения по ионной подвижности имеет линейную геометрию, инженеры Waters придали ему компактную циклическую форму. В ходе анализа ионы циркулируют в ионопроводе, все больше отдаляясь друг от друга. Исследователи могут контролировать разрешение ионов, задавая количество проходов, и детектировать изомерные соединения, которые невозможно разделить с помощью жидкостной хроматографии. Циклический ионопровод в сочетании с тандемной масс-спектрометрией предоставляет уникальные возможности для изучения ионной подвижности и структуры веществ.

$$
\because *
$$

Представленные новинки в области масс-спектрометрии расширяют возможности ученых в исследовании сложнейших природных объектов и открывают новые горизонты в медицине, фармацевтике и структурной химии. Следующая ежегодная конференция Американского массспектрометрического общества пройдет в Хьюстоне (США) с 31 мая по 4 июня 2020 года. 